\title{
Farm Animal Assisted Intervention with Individuals with Dementia: An Exploratory Study ${ }^{*}$
}

\author{
Julie Casey $^{1,2}$, Rick Csiernik ${ }^{2, *}$ \\ ${ }^{1}$ Nourishing Hearts Animal Assisted Therapy, Rodney, ON, Canada \\ ${ }^{2}$ Social Work Department, King's University College, London, ON, Canada
}

\begin{abstract}
An exploratory Animal Assisted Intervention study was conducted employing farm animals with individuals living with dementia living in a residential environment to assess if the intervention had any impact on behaviour. Fifteen individuals met the eligibility criteria but only 10 were able to participate in more than $67 \%$ of the sessions that consisted of petting, grooming and walking the animals; singing animal related songs, learning about the animal's history, species and breed; training the animal; reminiscing about past human-animal interactions and relationships; and reflecting on the animal's behaviours and story in relation to their own self. Individuals engaged in three one hour sessions for each of eight weeks. Outcomes were either positive or neutral with increased restful sleeping, decreases in verbally aggressive behaviour and depression and being less resistive to care was documented. The outcomes were sufficient to warrant a larger scale investigation working with farm animals and this population.
\end{abstract}

Keywords Animal Assisted Intervention, Canada, Dementia, Farm Animals

\section{Introduction}

Humans and animals have been evolving together through the domestication process that has occurred over the past ten thousand years. Human-animal interactions involve sensory, tactile and social engagement that stimulates the release of oxytocin; a hormone that changes our chemistry that has "yielded a relaxation that made our modern civilization possible" (Olmert, 2009, p. 180). It is only within the last 100 years that the majority of humans in the developed world have made a move away from regular contact with farm animals; and according to Olmert, we are the first society to disengage from animals in with our abrupt shift from farm to factory lifestyle following the
Industrial Revolution. However, this connection has not totally been lost within the therapeutic community with the rising interest in animal assisted intervention (AAI). Despite this attention relatively little is still empirically known about the impact of these interventions with research largely remaining anecdotal and descriptive (Stern \& Chur-Hansen, 2013). This includes studies where AAI was implemented in long term care facilities to help lessen the behavioural and psychological symptoms of dementia (BPSD) experienced by the elderly (Bernabei, De Ronchi, La Ferla, Moretti, Tonelli, et al, 2013).

Clinically significant neuropsychiatric symptoms are common in nursing home residents (Selbæk, Engedal, \& Bergh, 2013). BPSD includes aggression, wandering, restlessness, repeated actions, suspicion, apathy, sundowning and sexual behaviour (Alzheimer Society Canada, 2016). In 2014 Ontario Long Term Care Association (OLTCA) reported that 62\% of Ontario nursing home residents lived with Alzheimer's disease or other dementias while nearly one third had severe cognitive impairment. Since 2008 the proportion of residents with dementia increased by $6 \%$ while mild or moderate cognitive impairment rose by $8 \%$ with dual diagnosis of dementia coupled with a psychiatric diagnosis increasing $11 \%$. As well, one in three residents also had a psychiatric diagnosis such as anxiety, depression, bipolar disorder, or schizophrenia. This contributed to a $14 \%$ increase in moderately aggressive behaviour with nearly half of residents exhibiting some level of aggressive behaviour between 2010 and 2012. As the complexity of residents issues has increased so have incidents of aggressive behaviour which has led to concerns regarding the safety of both staff and residents.

AAI has been gaining greater acceptance and is now being utilized by a range of professions in diverse settings, including hospital critical care units, prisons, nursing homes, long-term care facilities, psychiatric institutions, hospice facilities, treatment centres and youth detention centers (Hanrahan, 2013). AAI supports individuals across the lifespan with diverse needs, including aging; and the ISSN: 2332-6840 (Online) 2332-6832 (Print) Copyright (C) 2018 by authors, all rights reserved. Authors agree that this article remains permanently open access under the terms of the Creative Commons Attribution License 4.0 International License 
full spectrum of mental health problems and symptoms that encompass emotional, cognitive, behavioural and social challenges, as well as dementia.. However, little has been written regarding if it has any merit for those suffering from dementia.

During this human-animal engagement production of the hormone oxytocin is increased (Beetz, Uvnäs-Moberg, Julius, \& Kotrschal, 2012). Oxytocin has widespread neurological, biological, emotional and social effects, including attachment, trust and social processing, while also decreasing anxiety, stress and aggression (Netherton \& Schatte, 2011). By stimulating the parasympathetic nervous system, oxytocin calms the fight-flight-freeze stress response, reducing the secretion of cortisol, aldosterone and adrenaline, while also increasing pain tolerance, lowering blood pressure, increasing vagal tone, decreasing inflammation, improving wound healing, facilitating learning, and lowering anxiety (Chandler, 2012). This has led to interest in examining AAI as a therapeutic mechanism to ameliorate the symptoms and impact of dementia for individuals living in long term care facilities.

Numerous AAI studies investigating the impact on BPSD have reported positive outcomes. Nordgren and Engstrom (2013) reported individuals experiencing dementia were filled with joy and well-being after engaging in AAI while Majic, Gutzmann, Heinz, Lang and Rapp (2013) stated AAI was "a promising option for treating symptoms of agitation/aggression and depression in elderly demented nursing home residents" (p. 1058). Richeson's (2003) pilot study tested the AAI intervention's effect on agitated behaviours and social interactions of older adults with dementia. Although the study was not randomized and the sample size was small, the outcomes indicated the AAI decreased agitation behaviours of persons with dementia as did the earlier work of Sellers (2006).

In a prospective, cluster randomized multi-centre trial ten nursing homes in Norway were randomized to either AAI with a dog or a control group with treatment as usual. Using a three-month post-intervention follow-up of both men and women aged 65 years or older, with a diagnosis of dementia or having a cognitive deficit a significant effect on depression and quality of life was found among the 28 participants in the experimental group compared to the 30 in the control group though no effects on agitation were reported (Olsen, Pedersen, Bergland, Enders - Slegers, Patil, \& Ihlebæk, 2016). In a related study 28 persons with dementia still living at home, along with 21 living in a residential care facility engaged in group animal-assisted activity. The participants interacted with a dog and its handler for 30 minutes, twice a week for 12 weeks leading to increased engagement in both groups (Olsen, Pedersen, Bergland, Enders-Slegers, \& Ihlebæk, 2016).

With the progression of dementia there is a lack of appropriate environmental, sensory and social stimulation which causes a simulation deprivation that leads to continued deterioration of motor and sensory skills. One response entails increased motor stimulation which are specific exercises to improve mobility and delay the decline of activities of daily living. AAI activities such as petting, grooming and walking of the intervention animal provide meaningful and motivating movements. Multisensory stimulation are activities that stimulate the senses without the need for higher cognition which helps to reduce behaviours and apathy while also increasing alertness and engagement. Again three is a role for AAI as human-animal interaction provides visual, olfactory, auditory and tactile stimulation through direct and indirect contact (Cruz, Marques, Barbosa, Figueiredo \& Sousa, 2011; Filan, \& Llewellyn-Jones, 2006; Sánchez, Millán-Calenti, Lorenzo-López, \& Maseda, 2013).

Reminiscence therapy according is the process of recalling personal experiences from the past and because with dementia there is a greater preservation of remote rather than recent memories; the act of reminiscing can improve function by "decreasing demands on impaired cognitive abilities and capitalize on the preserved ones" (Kim, Cleary, Hopper, Bayles, Mahendra \& Azuma, 2006, p. 1). AAI provides opportunity to stimulate remote memories of companion pets through talking, visual augmentative communication tools, as well as music and trivia activities.

Canada is historically a nation of farms. Many contemporary seniors grew up in settings surrounded by livestock including cows, pigs, sheep, goats, rabbits, chickens and other fowl. Even if individuals did not have a direct experience with farming, Olmert (2009) states, there is still a part within humans as a species, that wants to be back on the farm and this is evident in the increasing demand for "farm life" experience being offered to the public as theme parks. Although dogs are the most common intervention animal species (Chandler, 2012), there is no best animal to work with for AAI as different species bring different strengths (Mallon, 1994; Urichuk, Anderson, \& Chimo Project, 2003). It is dependent upon the therapist to identify and incorporate appropriate intervention animals into a treatment session. This perspective is supported by Chandler (2012) who stated "any farm animal is appropriate for intervention as long as it is healthy and not aggressive" (p. 72). There have been some studies that have used farm animals in AAI with individuals with a range of mental health issues including affective disoreders, anxiety, depression, and schizophrenia that have produced positive outcomes (Berget, Ekeberg, \& Braastad, 2008; Berget, Ekeberg, Pedersen, \& Braastad, 2011; Pedersen, Ihlebæk, \& Kirkevold, 2012; Pedersen, Nordaunet, Martinsen, Berget, \& Braastad, 2011) though none with individuals with dementia. This led us to undertake an exploratory study which asked what the impact was of conducting animal assisted intervention working with farm animals and 
individuals with dementia in a residential care setting.

\section{Methodology}

\section{Sample}

A specialized dementia wing of a long term care home in an urban Southwestern, Ontario city was selected for this exploratory study. The residential facility is home to 160 individuals, many of whom have a formal diagnosis of dementia and who are in the middle to late stages of this condition. Intervention animals used in this study were a sheep, rabbit, chickens and a goat. These animals had all previously worked with residents living in long term care homes with no previous incidents. To ensure the safety of the intervention animals, Animal Assisted Interventions International's standards of practice were followed for ethical human-animal practices and to protect the animals' well-being. The use of the Boat Inventory on Animal-Related Experiences (1994) was applied to reduce the risk of potential harm to the intervention animals. Additionally, the Guidelines for Wellness of Animals Involved, created by the International Association of Human-Animal Interaction Organizations were followed throughout the study to further ensure the wellbeing of the intervnetion animals (Lefebvre, Golab, Christensen, Castrodale, Aureden, et al, 2008).

To be included in the study participants needed to have had previous enjoyable experiences with animals as well as currently enjoying engaging with animals or expressing an interest in interacting with animals. Participants would need to be assessed as being able to benefit from reminiscent, motor and multisensory stimulation, while having no severe animal allergies, fears, or intense dislikes. In order to safe guard the animals participants with a history of perpetrating animal abuse were excluded as were any person with significant sensory deficits (being severely visually impaired or blind or having a severe auditory deficit or being deaf). As well, all participants had to be able to volunteer themselves or have their legal guardian(s) agree to allow the individual to participate in the study.

Informed consent from residents with legal capacity was obtained while informed consent from those without legal capacity was obtained from their family/guardian for the 15 individuals who met the inclusion criteria. This protocol was followed to ensure that there was no coercion or duress to participate nor that a resident's impaired cognitive abilities were used to include them in the study.

Of the 15 individuals who qualified for the study, 13 actively participated, three male and ten female with an average age 80.5 . Nine $(69.2 \%)$ of the participants were born in Canada, two (15.4\%) in the United Kingdom, with one $(7,7 \%)$ each in Germany and the United States. Ten, two male and eight female participated in $15(68.2 \%)$ or more of the 22 AAI sessions $($ mean $=19.2$, range $15-22)$ while three individuals, one male and two female participated in fewer than $50 \%$ (mean $=10.7$, range 10-11). Of the remaining two individuals one was transferred to another unit and one died prior to the completion of the post-treatment data collection. All participants had active engagement throughout their lives with animals, seven $(46.7 \%)$ with farm animals primarily though living or working on a farm but also having rabbits as pets while growing up.

\section{Instruments}

Demographic data including participant's age, sex, nationality, type of dementia, pet/animal history and connection to farming was collected. The information assisted in gaining a social understanding of who each resident was as well as identifying cultural and life experiences associated to animals and farming, while also screening for allergies, fears or risk. Six distinct quantitative instruments were also employed in this study.

\section{i Dementia Observational System (DOS) Tool}

The DOS tool is used to assess a person's behaviour over a 24-hour cycle to determine the occurrence, frequency, and duration of behaviours of concern. It is used upon admissions to establish a baseline behavioural profile, whenever there is a change or concern about the person's behaviours or to evaluate the effectiveness of a planned intervention on the care-plan that is addressing specific target behaviours. In this study the DOS was used to record behaviours in 60 minutes intervals using a numeric code:

1 - 2 sleeping in bed/sleeping in chair

3 awake/calm

4 noisy

5 restless / pacing

6 exit seeking

7 aggressive - verbal

8 aggressive - physical

9 resistive to care

10 physical aggression other than at care time (Hamilton, Harris, Le Clair, \& Collins, 2008).

\section{ii Aggressive Behaviour Scale (ABS)}

One of the more negative behaviours that arises as a result of dementia is aggressive behaviour. The ABS provides a measure of this behaviour. The ABS is strongly correlated with the Cohen Mansfield Agitation Inventory (1991) (correlation coefficient $=0.72, \mathrm{P}<.001$ ). It consists of four aggressive behaviour sub-scales: verbally abusive, physically abusive, socially inappropriate/disruptive behaviour, socially inappropriate/disruptive behaviour and resistance of care. The scale is scored 0-12 with greater scores indicating higher levels of aggressive behaviour. Alphas for the ABS were between 0.79 and 0.93 for samples from Ontario, Canada nursing homes and complex continuing care hospitals (Perlman, \& Hirdes, 2008). 
iii Changes in Health, End-Stage Disease and Signs and Symptoms (CHESS).

The nine item CHESS detects frailty and health instability and was designed to identify residents at risk of serious health decline. It is scored on a $0-5$ scale with higher scores associated with more severe outcomes including mortality. It is a composite measure addressing changes in health, end-stage disease, and symptoms and signs of medical problems. It is a strong predictor of mortality $(\mathrm{P}<.0001)$ independent of the effects of age, sex, activities of daily living impairment, cognition, and do-not-resuscitate orders. It is also strongly associated with physician activity, complex medical procedures, and pain $(\mathrm{P}<.001$ for each dependent variable) (Hirdes, Frijters, \& Teare, 2003).

\section{iv Cognitive Performance Scale (CPS)}

The CPS describes an individual's cognitive status. It has been validated against both the Mini-Mental State Examination (MMSE) and the Test for Severe Impairment. Its five scales are comatose, short-term memory, cognition skills for daily decision-making, expressive communication and eating. It is scored $0-6$ with higher scores indicating greater cognitive impairment (Morris, et al., 1994). The CPS showed substantial agreement with the MMSE in identifying cognitive impairment; sensitivity of $.94(95 \%$ CI. $.90, .98)$ with a diagnostic accuracy of .96 (95\% CI: .88, 1.0) (Hartmaier et al., 1995).

\section{v Depression Rating Scale (DRS)}

The DRS is a clinical depression scale scored 0-14 with a score of 3 or more indicating a potential or actual problem with depression. It has been validated against the Hamilton Depression Scale (94\% sensitivity) and the Cornell Scale for Depression in Dementia (78\% sensitivity) (Burrows, Morris, Simon, Hirdes, \& Phillips, 2000). It can be used to determine if there has been a change in affect among those with dementia.

\section{vi Revised Index of Social Engagement (RISE)}

The RISE describes an individual's sense of initiative and social involvement in their residential care facility. It was developed from the Index of Social Engagement (ISE), enhancing the reliability of the ISE by including additional dimensions of social engagement. This scale is scored 0-6 with higher scores indicating greater social involvement. It uses six indices to generate its total score (Gerritsen et al., 2008) to determine the degree to which an individual is active and social within an institution.

\section{Procedure}

As this was a beginning exploratory study a simple pre-post test design $(\mathrm{O} \times \mathrm{X})$ was utilized. The study consisted of a two-week period where baseline data was collected using the six instruments. This was followed by eight weeks of animal assisted intervention and a three-week post-intervention data collection period. Residents engaged in three one-hour group sessions each week during the study period. Activities consisted of petting, grooming and walking the animals; singing animal related songs, learning about the animal's history, species and breed; training the animal; reminiscing about past human-animal interactions and relationships; and reflecting on their perceived meaning of the animal's behaviour.

The team consisted of one counsellor with a Master of Social Work degree and intervention animals. As with any protocol involving both humans and animals there were inherent risks in this exploratory study. The risks were minimized through the participant screening process which assessed each volunteer for animal allergies and fears for particular animal species. Interaction with animals always poses a risk of zoonoses; disease transmission from humans from animals. To minimize this risk, all intervention animals were under veterinary care, were regularly thoroughly groomed, and had been vaccinated and dewormed. Hand hygiene policies were also followed throughout the eight-week study period.

The goal was to ascertain if this protocol produced changes in behaviour and affect among the residents with dementia.

\section{Ethics Approval}

Ethics approval to conduct the study was granted by the King's University College Research Ethics Review Committee.

\section{Results}

Table 1 provides a summary of the pre-post Dementia Observational System Results for the 13 participants who completed the study. However, before discussing the findings it is vital to note that the $z$-value and $p$ scores were calculated based upon weighted time. When data analysis began it was discovered that the DOS scores for each participant were incomplete. During the two week pre-test, 951 hours of activity was undocumented for the 13 participants, representing $21.8 \%$ of the time whereas for the three week post period 2,362 hours of the participants lives were not documented, representing $36.0 \%$ of their time. The number of hours for which no recordings were made is not inconsequential and thus the following discussion needs to be considered not only in light of it being an exploratory study but also with consideration for the incomplete data. This of course also brings into question whether the DOS is an appropriate tool for use in experimental research.

Statistically significant results were found on six of the nine variables. After having engaged with the intervention animals regardless of the number of sessions attended, the DOS scores indicated that participants slept more 
(behaviour 1-2), and were awake less (behaviour 3) compared to the two weeks prior to beginning AAI. As well, they created less noise (behavior 4), exhibited significantly less exit seeking (behavior 6), were less verbally aggressive (behaviour 7) and required less restive care (behavior 9).

Table 1. Dementia Observational System Results

\begin{tabular}{|c|c|c|c|c|}
\hline Behaviour & \multicolumn{4}{|c|}{ Average Weekly Time/Category (total hours) } \\
\hline & pre & post & z-value & p score \\
\hline $1-2$ & 82.2 & 107.4 & -3.1798 & $0.00148^{*}$ \\
\hline 3 & 55.6 & 40.2 & -2.9003 & $0.00374 *$ \\
\hline 4 & 1.1 & 0.6 & -2.7605 & $0.00578^{*}$ \\
\hline 5 & 13.3 & 12.7 & -0.06639 & 0.50926 \\
\hline 6 & 5.4 & 2.3 & -2.9191 & $0.0035^{*}$ \\
\hline 7 & 3.9 & 1.4 & -2.8304 & $0.00466^{*}$ \\
\hline 8 & 2.3 & 1.3 & -2.0616 & 0.0394 \\
\hline 9 & 3.4 & 1.4 & -2.7605 & $.00578^{*}$ \\
\hline 10 & 0.8 & 0.6 & -0.3844 & 0.70394 \\
\hline total & 168 & 168 & *statistically significant \\
\hline
\end{tabular}

Table 2 summarizes the pre-post results (mean scores) of the other five instruments. No significant differences were discovered in any of the scales.

Table 2. Pre-Post Mean Instrument Scores

\begin{tabular}{|c|c|c|c|c|}
\hline & $\begin{array}{c}\text { Pre-test } \\
\text { mean }\end{array}$ & $\begin{array}{c}\text { Post-Test } \\
\text { mean }\end{array}$ & z-value & p score \\
\hline $\begin{array}{c}\text { Aggressive } \\
\text { Behavior }\end{array}$ & 1.8 & 1.7 & -1.0225 & 0.30772 \\
\hline $\begin{array}{c}\text { Changes in } \\
\text { Health, End } \\
\text { Stage } \\
\text { Disease \& } \\
\text { Signs \& } \\
\text { Symptoms }\end{array}$ & 1.0. & 1.1 & -2.4111 & 0.01596 \\
\hline $\begin{array}{c}\text { Cognitive } \\
\text { Performance } \\
\text { Scale }\end{array}$ & 4.0. & 3.9 & -0.7135 & 0.4777 \\
\hline $\begin{array}{c}\text { Depression } \\
\text { Rating Scale }\end{array}$ & 2.9 & 2.2 & -2.4809 & 0.01314 \\
\hline $\begin{array}{c}\text { Index of } \\
\text { Social } \\
\text { Engagement }\end{array}$ & 2.6 & 2.8 & -1.5689 & 0.11642 \\
\hline
\end{tabular}

\section{Discussion}

With the aging population and rise in dementia rates, long term care facilities are seeking alternatives to pharmacological interventions as a healthier, safer and more cost effective approach to managing responsive behaviours which include aggression, wandering, restlessness, and repeated negative actions. This exploratory study examined the impact of farm animal assisted intervention as a means to reduce these responsive behaviours.. Despite increasing research regarding the beneficial impact of animals on human physical and mental health, these benefits are not fully understood as the majority of research remains anecdotal and descriptive (Stern \& Chur-Hansen, 2013). As well, since the AAI field is dominated by canine animals, this study also introduces the potential of farm animal species as intervention animals, especially with the older generation with a historical connection and life experience with farming.

This exploratory study a pre-test, post-test design produced mixed results. No statistically significant pre-post differences were observed with regards to aggressive behaviour, changes in health status, cognitive performance nor social engagement. The Dementia Observational System Results protocol did however highlight some positive pre-post differences in the areas of sleep, wakefulness, and exit seeking along with between group differences in the areas of verbally aggressive behaviour and restive care. However, there was no regression in any area of the study by participants. While there were not significant improvements in all areas, given the progressive nature of dementia, preserving function and quality of life can in and of itself be considered success. The presence of the farm animals produced some positive results in a short period of time while not producing any negative outcomes. However, there were distinct limitations with the research. As with any exploratory study attempting to determine if a new intervention has merit, a major limitation was the use of a simple one group pre-post design with no comparison group. As well, the small sample size and that by using a convenience sampling model all participants came from one facility in one community is a limiting factor. Another limitation of the study arose due to gaps in reporting using the Dementia Observational System as this was the primary data collection instrument. Over $20 \%$ of the pre-test time was not recorded while one third of the post-test observations were not made forcing weighted time to be used in the calculations. As well, one of the participants died during the post-treatment period and it is impossible to determine what if any impact this had on the other members of the study.

The potential shown in the outcomes and the limits that can be readily overcome justify repeating the study with a larger sample in multiple institutions with more precise measurement instruments. Other factors to consider in moving forward in this area of research are how frequent the visits should be, if additional animal contact would provide more positive outcomes and if the study period needs to be extended. As well, the phenomenon of sundowning, was not taken into consideration and could have been a confounding variable that needs to be considered in future research. As the study of responsive behaviours is becoming an important area of investigation with individuals who have dementia greater attention to elements of well-being such as depression are also an 
avenue for future AAI research.

\section{REFERENCES}

Alzheimer Society Canada. (2016). Understanding Behaviours. Retrieved fromhttp://www.alzheimer.ca/en/Living-with-dementi a/Understanding-behaviour

Animal Assisted Interventions International. (2015). Standards of Practice for Animal Assisted Interventions: Animal Assisted Therapy. Retrieved from http://www.aai-int.org/wp-content/uplo ads/2016/02/AAI-Standards-final.pdf

Bernabei, V., De Ronchi, D., La Ferla, T., Moretti, F., Tonelli, L., Ferrari, B.. . Atti, A. R. (2013). Animal-assisted interventions for elderly patients affected by dementia or psychiatric disorders: A review. Journal of Psychiatric Research, 47(6), 762-773.

Beetz, A., Uvnäs-Moberg, K., Julius, H., \& Kotrschal, K. (2012). Psychosocial and psychophysiological effects of human-animal interactions: the possible role of oxytocin. Frontiers in psychology, 3 (234). Doi 10.3389/fpsyg.2012.00234

Berget, B., Ekeberg, Ø., \& Braastad, B. (2008). Animal-assisted therapy with farm animals for persons with psychiatric disorders: effects on self-efficacy, coping ability and quality of life, a randomized controlled trial. Clinical practice and epidemiology in mental health, 4(1), 9. Doi: 10.1186/1745-0179-4-9

Berget, B., Ekeberg, Ø., Pedersen, I., \& Braastad, B. (2011) Animal-assisted therapy with farm animals for persons with psychiatric disorders: effects on anxiety and depression, a randomized controlled trial. Occupational Therapy in Mental Health, 27(1), 50-64.

Boat, B. (1994). Boat inventory on animal-related experiences. Cincinnati, OH: University of Cincinnati, Department of Psychiatry.

Burrows, A., Morris, J., Simon, S., Hirdes, J., \& Phillips, C. (2000). Development of a minimum data set-based depression rating scale for use in nursing homes. Age and ageing, 29(2), $165-172$

Chandler, C. K. (2005). Animal assisted therapy in counseling. New York: Routledge.

Cohen-Mansfield, J. (1991). Instruction manual for the Cohen-Mansfield agitation inventory (CMAI). Research Institute of the Hebrew Home of Greater Washington.

Cruz, J., Marques, A., Barbosa, A. L., Figueiredo, D., \& Sousa, L. (2011). Effects of a motor and multisensory-based approach on residents with moderate-to-severe dementia. American Journal of Alzheimer's Disease and Other Dementias, 26(4), 282-289.

Filan, S. L., \& Llewellyn-Jones, R. H. (2006). Animal-assisted therapy for dementia: A review of the literature. International Psychogeriatrics, 18(4), 597-611.

Gerritsen, D., Steverink, N., Frijters, D., Hirdes, J., Ooms, M., \& Ribbe, M. (2008). A revised Index for Social Engagement for long-term care. Journal of Gerontological Nursing, 34(4), 40-48.

Hamilton, P, Harris, D., Le Clair, K., \& Collins, J. (2008). Putting the P.I.E.C.E.S. Together: A Model for Collaborative Care and Changing Practice. A Learning Resource for Professionals Providing Long-Term Care to Older Adults with Complex
Physical and Cognitive/Mental Health Changes" 6th Edition (R). Toronto: P.I.E.C.E.S

Hanrahan, C., (2013). Social work and human animal bonds and benefits in health research: A provincial study. Critical Social Work, 14(1). Retrieved from http://www1.uwindsor.ca/criticalso cialwork/SWhumananimalbonds

Hartmaier, S., Sloane, P., Guess, H., Koch, G., Mitchell, C., \& Phillips, C. (1995). Validation of the minimum data set cognitive performance scale: Agreement with the mini-mental state examination. The Journals of Gerontology Series A: Biological Sciences and Medical Sciences, 50(2), M128-M133.

Hirdes, J., Frijters, D., \& Teare, G. (2003).The MDS CHESS

Scale: A new measure to predict mortality in the institutionalized elderly. Journal of the American Geriatrics Society, 51(1), 96100 .

International Association of Human-Animal Interaction Organizations(IAHAIO). (2014). Definitions for animal assisted Intervention and guidelines for wellness of animals involved. Retrieved fromhttp://iahaio.org/new/fileuploads/4163IAHAIO $\%$ 20WHITE\%20PAPER-\%20FINAL\%20-\%20NOV\%2024-2014. pdf

Kim, E. S., Cleary, S.J., Hopper, T., Bayles, K. A., Mahendra, N., Azuma, T., \& Rackley, A. (2006). Evidence-based practice recommendations for working with individuals with dementia: Group reminiscence therapy. Journal of Medical Speech-Language Pathology, 14(3), xxiii-xxxiv.

Lefebvre, S. L., Golab, G. C., Christensen, E., Castrodale, L., Aureden, K., Bialachowski, A.. . Writing Panel of Working Group. (2008). Guidelines for animal-assisted interventions in health care facilities. AJIC: American Journal of Infection Control, 36(2), 78-85.

Majić, T., Gutzmann, H., Heinz, A., Lang, U. E., \& Rapp, M. A (2013). Animal-assisted therapy and agitation and depression in nursing home residents with dementia: A matched case-control trial. The American Journal of Geriatric Psychiatry : Official Journal of the American Association for Geriatric Psychiatry, 21(11), 1052-1059.

Mallon, G. (1994). Cow as co-therapist: Utilization of farm animals as therapeutic aides with children in residential treatment. Child \& Adolescent Social Work Journal, 11(6), 455-474.

Morris, J., Fries, B., Mehr, D., Hawes, C., Phillips, C., Mor, V., \& Lipsitz, L. (1994). MDS cognitive performance scaleC. Journal of gerontology, 49(4), M174-M182.

Netherton, E., \& Schatte, D. (2011). Potential for oxytocin use in children and adolescents with mental illness. Human Psychopharmacology: Clinical and Experimental, 26(4-5), 271-281.

Nordgren, L., \& Engström, G. (2014). Animal-assisted intervention in dementia: Effects on quality of life. Clinical Nursing Research, 23(1), 7.

Olmert, M. D. (2009). Made for each other: The biology of the human-animal bond. Cambridge, MA: Lifelong Books/Da Capo Press.

Olsen, C., Pedersen, I., Bergland, A., Enders-Slegers, M. J., \& Ihlebæk, C. (2016). Engagement in elderly persons with dementia attending animal-assisted group activity. Dementia, doi: 1471301216667320 
Olsen, C., Pedersen, I., Bergland, A., Enders - Slegers, M. J., Patil, G., \& Ihlebæk, C. (2016). Effect of animal - assisted interventions on depression, agitation and quality of life in nursing home residents suffering from cognitive impairment or dementia: a cluster randomized controlled trial. International journal of geriatric psychiatry, 31(12), 1312-1321.

Ontario Long Term Care Association. (2014). This is Long Term Care. Retrieved from http://www.oltca.com/oltca/Documents/Re ports/This_is_LongTerm_Care_2014_Final.pdf

Perlman, C. \& Hirdes, J. (2008). The aggressive behavior scale: a new scale to measure aggression based on the minimum data set. Journal of the American Geriatrics Society, 56(12), 2298-2303.

Pedersen, I., Ihlebæk, C., \& Kirkevold, M. (2012). Important elements in farm animal-assisted interventions for persons with clinical depression: a qualitative interview study. Disability and rehabilitation, 34(18), 1526-1534.

Pedersen, I., Nordaunet, T., Martinsen, E. W., Berget, B., \& Braastad, B. (2011). Farm animal-assisted intervention: Relationship between work and contact with farm animals and change in depression, anxiety, and self-efficacy among persons with clinical depression. Issues in mental health nursing, 32(8), 493-500.

* This study was supported by and completed with the assistance of McCormick Dementia Research, London, Ontario
Richeson, N. (2003). Effects of animal-assisted therapy on agitated behaviors and social interactions of older adults with dementia. American journal of Alzheimer's disease and other dementias, 18(6), 353-358.

Sánchez, A., Millán-Calenti, J. C., Lorenzo-López, L., \& Maseda, A. (2013). Multisensory stimulation for people with dementia: a review of the literature. American Journal of Alzheimer's Disease \& Other Dementias, 28(1), 7-14.

Selbæk, G., Engedal, K., \& Bergh, S. (2013). The prevalence and course of neuropsychiatric symptoms in nursing home patients with dementia: A systematic review. Journal of the American Medical Directors Association, 14(3), 161-169.

Sellers, D. M. (2006). The evaluation of an animal assisted therapy intervention for elders with dementia in Long-term care. Activities, Adaptation \& Aging, 30(1), 61-77.

Stern, C., \& Chur-Hansen, A. (2013). Methodological considerations in designing and evaluating animal-assisted interventions. Animals: An Open Access Journal from MDPI, 3(1), $127-141$.

Urichuk, L. J., Anderson, D., \& Chimo Project. (2003). Improving mental health through animal-assisted therapy. Edmonton, Alberta: Chimo Project. 